\title{
3D Brachytherapy Treatment in Locally Advanced Cervical Cancer: A Case Presentation
}

\author{
(D) Kamuran İBiş, ${ }^{1}$ (D) Nazmiye DÖNMEZ KESEN² \\ 'Department of Radiation Oncology, Istanbul University, Institute of Oncology, Istanbul-Turkey \\ ${ }^{2}$ Department of Medical Physics, Istanbul University, Institute of Oncology, Istanbul-Turkey
}

\begin{abstract}
SUMMARY
Cervical cancer is the most frequent gynecologic malignancy worldwide and is the fourth most common cancer among women. The treatment of locally advanced cervical cancer is concomitant radiochemotherapy. Intracavitary brachytherapy is used following concomitant external radiotherapy with cisplatin chemotherapy. Three-dimensional brachytherapy is currently the treatment of choice for cervical cancer. It was previously shown that high-dose rate (HDR) brachytherapy and low-dose rate brachytherapy have similar survival rates in cervical cancer. The advantages of HDR brachytherapy are possibility of treatment in outpatient basis, no radiation exposure for staff, advanced ability of controlling the immobilization of applicators, and better dose optimization. In this case report, the treatment planning of a patient with locally advanced cervical cancer from Istanbul University Oncology Institute, with three-dimensional planning and HDR intracavitary brachytherapy following chemoradiotherapy, is discussed.
\end{abstract}

Keywords: Brachytherapy; cervical cancer; radiotherapy.

Copyright $\odot$ 2019, Turkish Society for Radiation Oncology

\section{Introduction}

Cervical cancer is the most frequent gynecologic malignancy worldwide and is the fourth most common cancer among women. [1] More than $90 \%$ of cervical tumors are squamous cell carcinomas, whereas 7\%-10\% of tumors are adenocarcinomas. Magnetic resonance imaging (MRI) is used for the initial evaluation, as well as for the evaluation, of extracervical spread of the tumor.[2] MRI is also useful for brachytherapy treatment planning and precise target volume definition. [3] 2-[18F]-fluoro-2-deoxy-D-glucose (FDG) positron emission tomography (PET)-computed tomography (CT) is increasingly used for the determination of treatment response in addition to diagnosis.[4,5]

The Federation Internationale de Gynecologie et d'Obstetrique (FIGO) staging system was based prin- cipally on clinical examination. It was revised in 2018 by the FIGO Gynecologic Oncology Committee, and imaging and pathological findings were added if available.[6] Radiological imaging, especially MRI and PET-CT scanning, occurred for staging near treatment planning and response evaluation. Patients with locally advanced cervical cancer (stages IB2 to IVA) are treated with brachytherapy following external radiotherapy (RT) combined with chemotherapy (cisplatin).

We present a case with cervical cancer treated with brachytherapy following concomitant radiochemotherapy in the Department of Radiation Oncology of the Institute of Oncology at Istanbul University.

\section{Case Report}

A 71-year-old woman suffering from vaginal hemorrhage for 2 months was admitted to our clinic. The pa- 
tients' medical history, as well as her family history, was unremarkable. The ages of menarche and menopause were 14 and 35 years, respectively. The histopathological
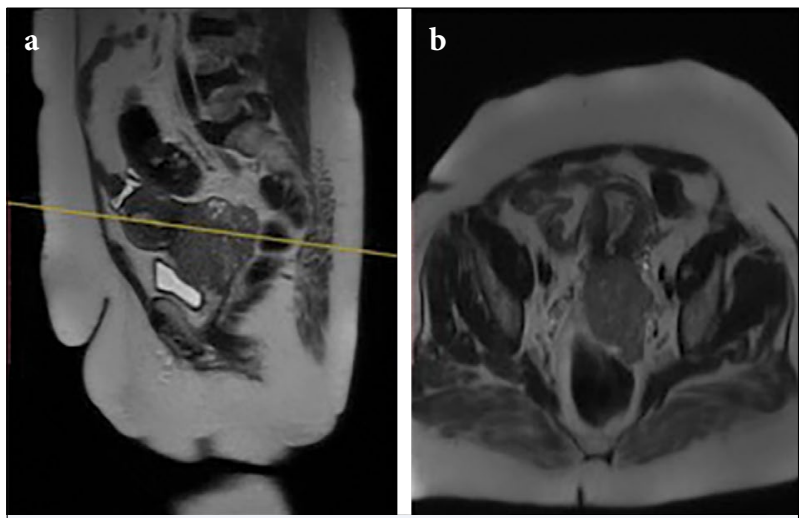

Fig. 1. Abdominopelvic MRI (T2-weighted images) prior to treatment. (a) Sagittal and (b) transverse images. evaluation of the biopsy obtained from gynecologic oncology revealed squamous cell carcinoma of the cervix. Bimanual rectovaginal examination prior to the treatment detected eroded cervix by endophytic cervical tumor and left parametrial invasion. Abdominopelvic MRI showed a $73 \times 65 \mathrm{~mm}$ sized cervical tumor that invaded the left parametrium (Fig. 1). Pretreatment PETCT evaluation revealed a $54 \times 55 \mathrm{~mm}$ sized mass with increased FDG uptake in the cervical region (Fig. 2). Intracavitary brachytherapy following external pelvic RT with concomitant chemotherapy was planned for the patient classified as stage IIB according to the FIGO 2018 staging system. The patient received $50 \mathrm{~Gy} / 25 \mathrm{Fr}$ external RT (three-dimensional conformal radiation therapy), and concomitant cisplatin of $40 \mathrm{mg} / \mathrm{m}^{2}$ was also delivered. Bimanual rectovaginal examination following external beam RT revealed dramatic regression of cervical lesion and only mild left parametrial invasion. Abdominopelvic MRI prior to brachytherapy
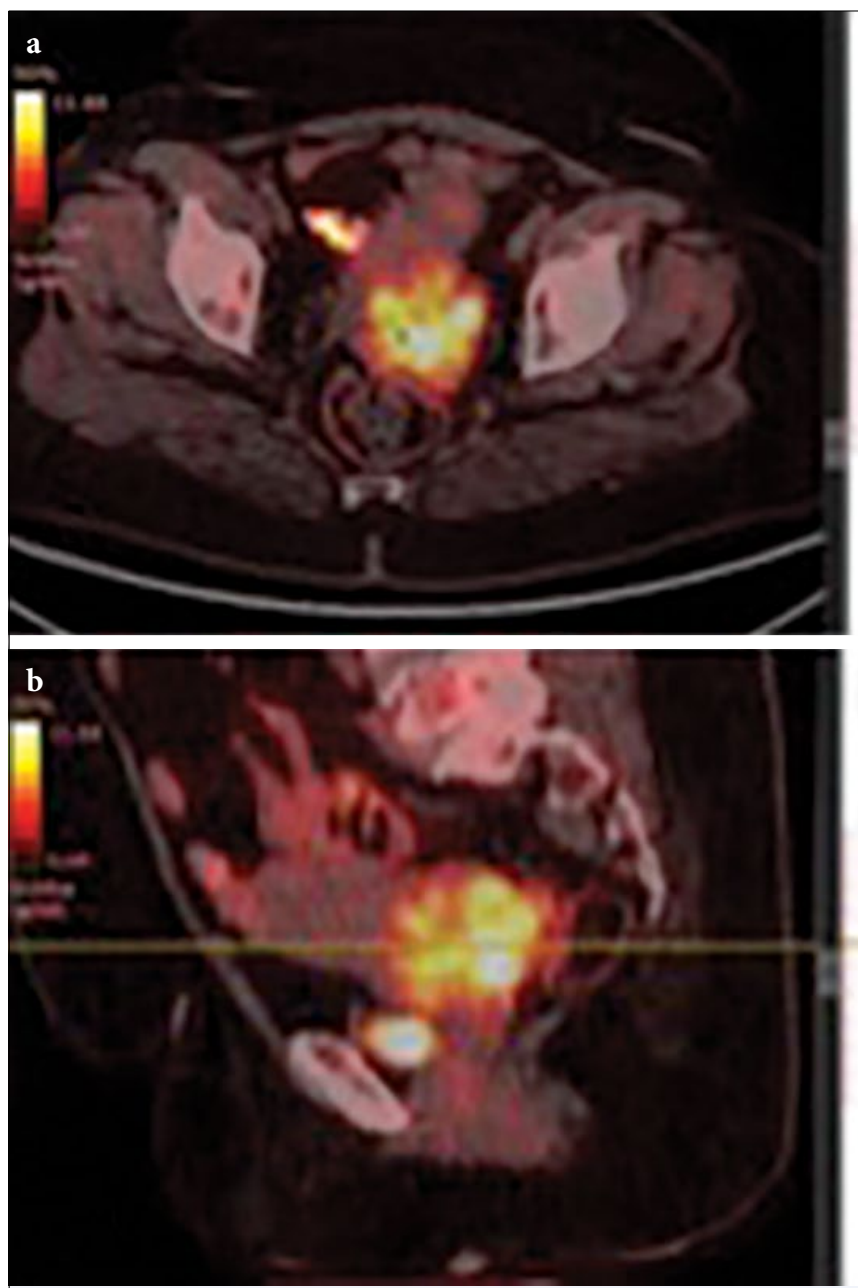

Fig. 2. PET-CT images prior to treatment. (a) Sagittal and (b) transverse images. 

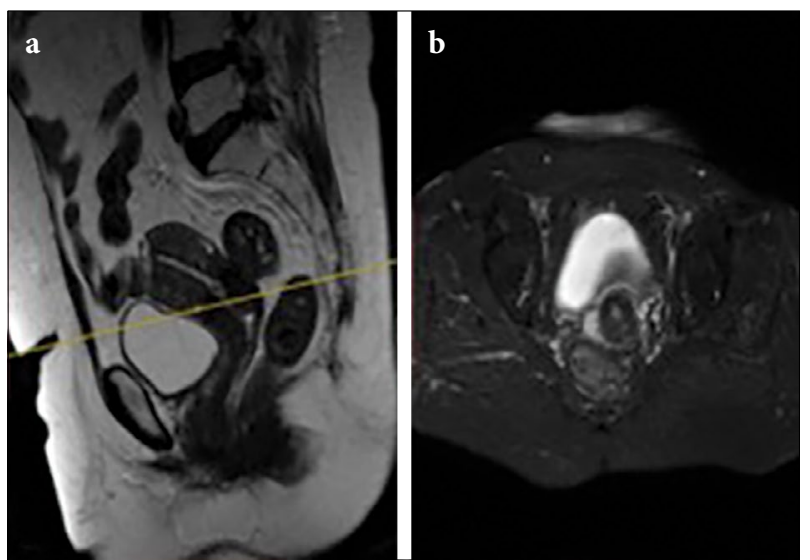

Fig. 3. Abdominopelvic MRI (T2-weighted images) following external radiotherapy. (a) Sagittal and (b) transverse images.
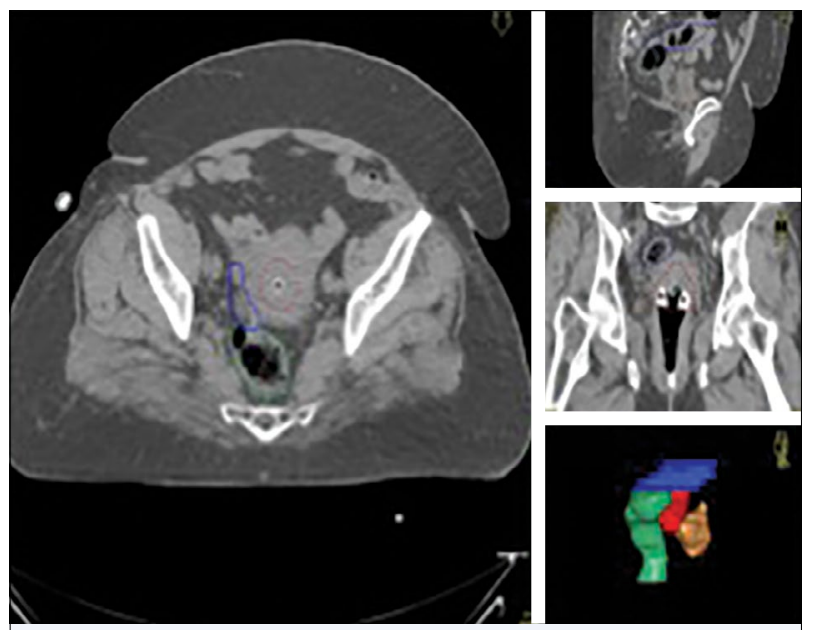

Fig. 4. Target volume and critical organ volumes in axial, coronal, and sagittal planes.

showed complete response of the cervical lesion (Fig. 3). The patient was prepared for general anesthesia before brachytherapy. She was examined under general anesthesia. Urinary catheter was inserted, and silver seed was also placed into the cervix. The most suitable applicators were selected for the patient. Thereafter, a $26 \mathrm{~mm}$ diameter ring applicator was placed following insertion of a $4 \mathrm{~cm}$ length tandem. Anteroposterior and lateral radiographs were obtained via $\mathrm{C}$-arm X-ray machine. The patient was scanned with a $2 \mathrm{~mm}$ slice thickness by Philips Brilliance Big Bore 4D CT machine. Images from CT scan were imported into the brachytherapy planning system (Oncentra version 4.0; Nucletron, Veenendaal, Netherlands). Then, CT images were contoured for high-risk clinical target volume (HR-CTV) and organs at risk (bladder, rectum, and sigmoid) using the Groupe Européen de Curiethérapie-European Society for Therapeutic Radiation Oncology (GEC-ESTRO) guidelines (Fig. 4).

At the beginning of brachytherapy treatment planning, catheter reconstruction was done directly on CT to determine the path for the source to track in each catheter. The offset and indexer values of the applicators were entered into the planning system $(-7 \mathrm{~mm}$ and $-6 \mathrm{~mm}$ offset values for ring and tandem applicators, respectively; $1500 \mathrm{~mm}$ indexer value for each applicator). Figure 5 shows the catheter reconstruction on CT images for different planes.

In the planning system, ensure that each catheter has the same number as the device channel number. After catheter reconstruction, the normalization process was performed. Target volume was selected for normalization point. Plan was normalized such that $90 \%$ of the target volume received the prescribed dose.

The source dwell positions were automatically generated by providing a $1-2 \mathrm{~mm}$ margin to the target volume. After autoactivation of the source dwell positions, the dose distribution was controlled in each CT cross-section. The source dwell positions were created manually by using the Forward Optimization Method (FOM) to achieve optimal target coverage with less critical organ doses. FOM is one of the treatment plan optimization methods in which treatment plans are generated manually. In this method, dwell positions
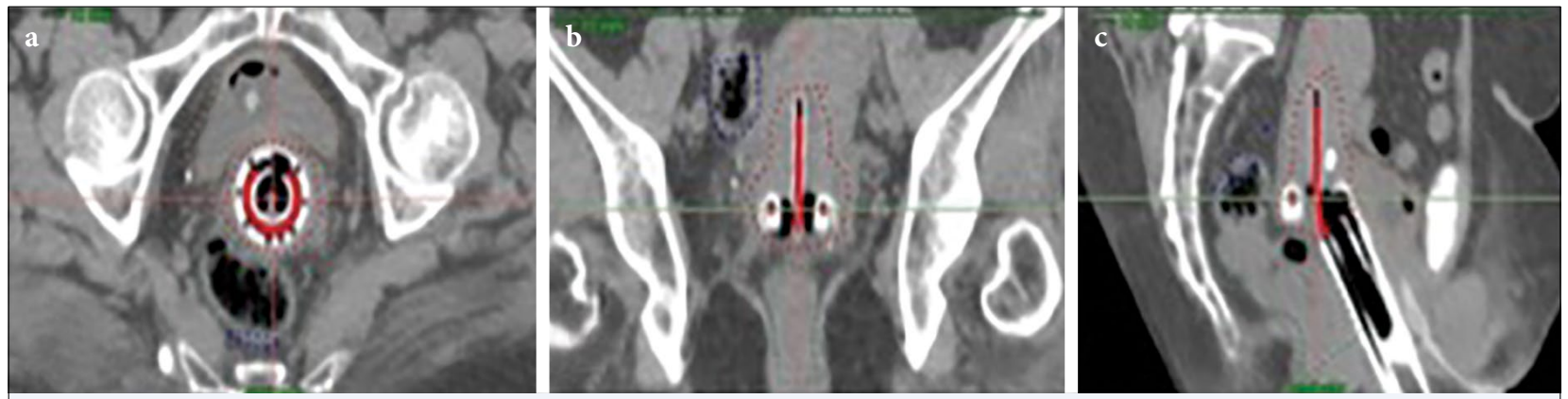

Fig. 5. Catheter reconstruction view from (a) transverse, (b) coronal, and (c) sagittal images. 

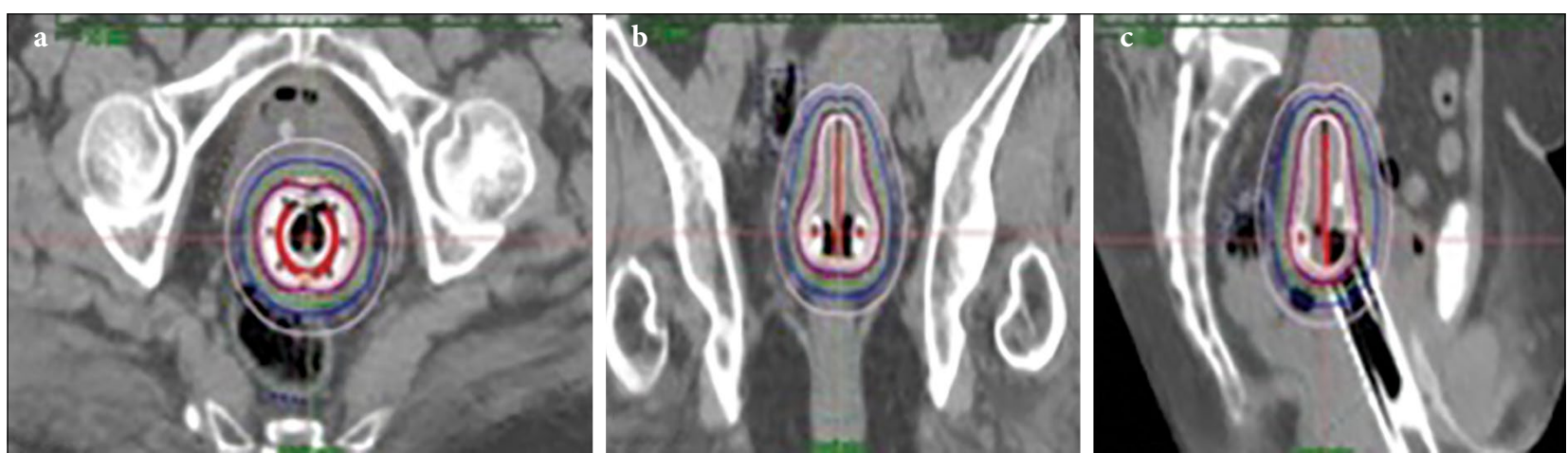

Fig. 6. Treatment plan dose distributions in (a) transverse, (b) coronal, and (c) sagittal planes.

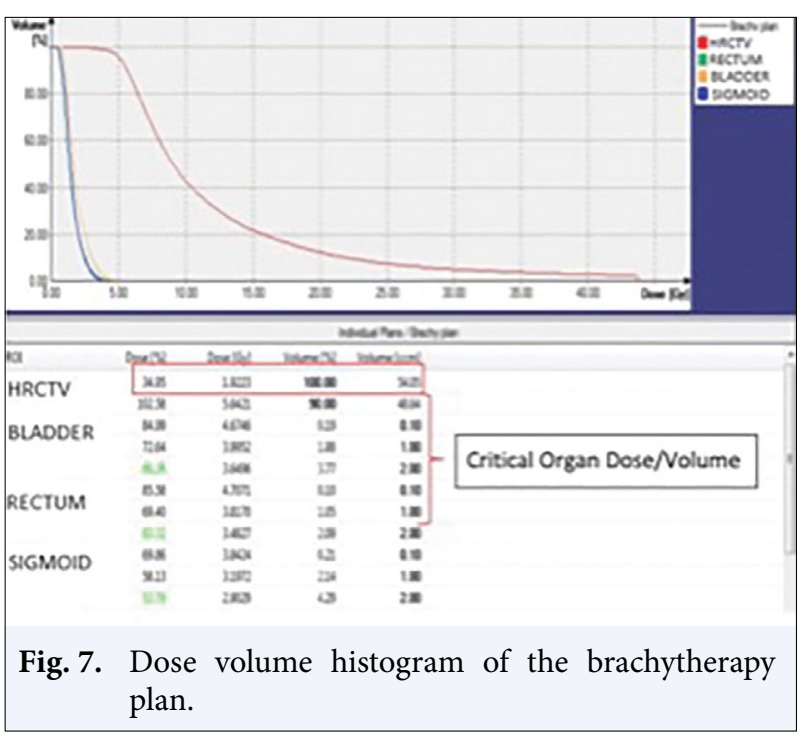

and dwell times are changed manually until the desired dose distribution on the target volume is achieved without exceeding the critical organ dose limits. [7]

The prescribed dose was obtained by using biological equivalent dose models. The dose prescribed that $90 \%$ of the target volume received 5.5 Gy per fraction, and that the plan was performed in five fractions. Graphical dose optimization was conducted by changing the isodoses manually to maximize target volume conformality and minimize critical organ doses (Fig. 6). This process continues until the appropriate dose distribution is achieved by controlling the dose volume histogram (DVH). DVH of the treatment plan is shown in Figure 7. Great care was taken to retain the target volume of the cold dose regions and the hot dose regions in the rectum, bladder, and sigmoid and $2 \mathrm{cc}, 1$ $\mathrm{cc}$, and $0.1 \mathrm{cc}$ volumes within the specified limits.

The gynecologic examination 2 months after treatment was found to be normal. Month 3 MRI scan and also month 6 PET-CT scan showed complete response.
The patient remains disease-free for 4 years following the initial treatment.

\section{Discussion}

Brachytherapy following concomitant radiochemotherapy is the standard treatment in locally advanced cancer of the cervix. Treatment planning of brachytherapy through three-dimensional imaging accurately defines the tumor and aids with the accuracy of irradiation dose delivery that may also reduce normal tissue doses and toxicity.[8] The GEC-ESTRO guidelines $[9,10]$ describe volumes for MR-based contouring. The American Brachytherapy Society organized a study about the use of image-based brachytherapy in 2007 [11] and updated it in 2014. A panel of radiation oncologists specializing in gynecologic oncology established a contouring atlas to delineate the CTV in locally advanced cancer of the cervix with a high consensus rate. Patients with parametrial extension mostly gained advantage from MRI scanning with respect to delicate brachytherapy planning. [12] The atlas is available online at www.nrgoncology.org. It is recommended that $90 \%$ of HR-CTV dose-planning within the EQD2 limits (EQD2 $\geq 80 \mathrm{~Gy}$ ) for D2cc should be 90 Gy for the bladder and 70-75 Gy for the rectum and sigmoid colon. The fraction doses of brachytherapy were also determined according to the BED and EQD2 dose calculations in our treatment planning. The GECESTRO guidelines delineated $3 \mathrm{D}$ planning volumes in brachytherapy in 2005 and determined the tumor and whole cervix tissue as HR-CTV while brachytherapy and recommended total delivery of the prescribed dose to HR-CTV. Intermediate risk CTV (IR-CTV) was defined as a $1 \mathrm{~cm}$ margin around HR-CTV+ initial sites of involvement. [9] It is used less frequently than HR-CTV in the USA. HR-CTV does not contain endometrium and vagina unless an obvious residual disease initially. Eifel and Klopp believed that regression of the vaginal 
or myometrial infiltration area toward the cervix is unlikely in contrast to paracervical tumors. Therefore, they suggested including the initial site of disease into the HR-CTV especially if it is not used in IR-CTV treatment planning.[13] The IR-CTV was not used in our clinic. We routinely include the initial site of disease into the HR-CTV if uterus and/or vaginal extension are present at the initial evaluation. Although it is possible to perform adaptive brachytherapy using simulation CT scan at the beginning of each fraction of brachytherapy, it is not a very practical approach in daily routine in most $\mathrm{RT}$ clinics. Similar to the presented case, we normally perform the remaining fractions in patients with complete response if the organ at risk doses are acceptable, after placing the seed into the cervix, and confirming the positions of applicators with the aid of anteroposterior and lateral radiographs. We prefer scanning with simulation CT for each brachytherapy application if there is residual tumor or need for re-check of organ at risk doses.

\section{Conclusion}

Brachytherapy is an important treatment modality in locally advanced cervical cancer. 3D brachytherapy provides high local control rates with maximum protection of the organs under risk in the course of irradiation.

Informed consent: The patient allowed the use of medical data and images for the publication as a case report.

Peer-review: Externally peer-reviewed.

Conflict of Interest: The authors declare that they have no conflict of interest.

Authorship contributions: Concept - K.I..; Design - K.İ., N.D.K.; Supervision - K.İ.; Materials - K.İ., N.D.K.; Data collection \&/or processing - K.I., N.D.K.; Analysis and/or interpretation - K.I..; Literature search - K.I..; Writing - K.I.., N.D.K.; Critical review - K.İ.

\section{References}

1. World Health Organization. Globocan 2012: Estimated Cancer incidence, mortality and prevalence worldwide in 2012. Available at: http://globocan.iarc. fr/Pages/fact_sheets_cancer.aspx. Accessed June 21, 2019.

2. Hricak H, Powell CB, Yu KK, Washington E, Subak LL, Stern JL, et al. Invasive cervical carcinoma: role of MR imaging in pretreatment work-up--cost minimization and diagnostic efficacy analysis. Radiology 1996;198(2):403-9.

3. Dimopoulos JC, Schard G, Berger D, Lang S, Goldner G, Helbich T, et al. Systematic evaluation of MRI findings in different stages of treatment of cervical cancer: potential of MRI on delineation of target, pathoanatomic structures, and organs at risk. Int J Radiat Oncol Biol Phys 2006;64(5):1380-8.

4. Schwarz JK, Grigsby PW, Dehdashti F, Delbeke D. The role of 18F-FDG PET in assessing therapy response in cancer of the cervix and ovaries. J Nucl Med 2009;50 Suppl 1:64S-73S.

5. Schwarz JK, Lin LL, Siegel BA, Miller TR, Grigsby PW. 18-F-fluorodeoxyglucose-positron emission tomography evaluation of early metabolic response during radiation therapy for cervical cancer. Int J Radiat Oncol Biol Phys 2008;72(5):1502-7.

6. Bhatla N, Aoki D, Sharma DN, Sankaranarayanan R. Cancer of the cervix uteri. Int J Gynaecol Obstet 2018;143 Suppl 2:22-36.

7. Elodie R, Chung MT. Optimization methods for high dose rate brachytherapy treatment planning. Master of Applied Science, Graduate Department of Mechanical and Industrial Engineering University of Toronto; 2016.

8. Petric P, Pötter R, Van Limbergen E, Haie-Meder C. Adaptive contouring of the target volume and organs at risk. In: Viswanathan AN, Kirisits C, Erickson B, Potter R, eds. Gynecologic radiation therapy: Novel approaches to image-guidance and management. Berlin, Germany: Springer; 2011. p. 99-118.

9. Haie-Meder C, Pötter R, Van Limbergen E, Briot E, De Brabandere M, Dimopoulos J, et al. Recommendations from Gynaecological (GYN) GEC-ESTRO WorkingGroup (I): concepts and terms in 3D image based 3D treatment planningin cervix cancer brachytherapy with emphasis on MRI assessment of GTV and CTV. Radiother Oncol 2005;74(3):235-45.

10. Pötter R, Dimopoulos J, Kirisits C, Lang S, HaieMeder C, Briot E, et al. Recommendations for image-based intracavitary brachytherapy of cervix cancer: the GYN GEC ESTRO Working Group point of view: in regard to Nag et al. (Int J Radiat Oncol Biol Phys 2004;60:1160-1172). Int J Radiat Oncol Biol Phys 2005;62(1):293-5.

11. Viswanathan AN, Erickson BA. Three-dimensional imaging in gynecologic brachytherapy: a survey of the American Brachytherapy Society. Int J Radiat Oncol Biol Phys 2010;76(1):104-9.

12. Viswanathan AN, Erickson B, Gaffney DK, Beriwal S, Bhatia SK, Lee Burnett O 3rd, et al. Comparison and consensus guidelines for delineation of clinical target volume for CT- and MR-based brachytherapy in locally advanced cervical cancer. Int J Radiat Oncol Biol Phys 2014;90(2):320-8.

13. Eifel P, Klopp AH. Brachytherapy. In: Eifel P, Klopp AN, eds. Gynecologic radiation Oncology: A Practical Guide. Wolters Kluwer; 2017. p. 113-31. 